\title{
Design and methods of the NiCK study: neurocognitive assessment and magnetic resonance imaging analysis of children and young adults with chronic kidney disease
}

\author{
Erum A Hartung ${ }^{1,2}$, Nina Laney ${ }^{1}$, Ji Young Kim³ ${ }^{3}$, Rebecca L Ruebner ${ }^{1,2}$, John A Detre ${ }^{4}$, Hua-Shan Liu ${ }^{5,6}$, \\ Christos Davatzikos ${ }^{7}$, Guray Erus ${ }^{7}$, Jimit J Doshi ${ }^{7}$, Robert T Schultz ${ }^{2,8,9}$, John D Herrington ${ }^{8,9}$, Abbas F Jawad ${ }^{2,10}$, \\ Divya G Moodalbail ${ }^{11}$, Ruben C Gur ${ }^{9,12}$, Allison M Port ${ }^{12}$, Jerilynn Radcliffe ${ }^{2,13}$, Stephen R Hooper ${ }^{14}$ and Susan L Furth ${ }^{1,2,15^{*}}$
}

\begin{abstract}
Background: Chronic kidney disease is strongly linked to neurocognitive deficits in adults and children, but the pathophysiologic processes leading to these deficits remain poorly understood. The NiCK study (Neurocognitive Assessment and Magnetic Resonance Imaging Analysis of Children and Young Adults with Chronic Kidney Disease) seeks to address critical gaps in our understanding of the biological basis for neurologic abnormalities in chronic kidney disease. In this report, we describe the objectives, design, and methods of the NiCK study.

Design/methods: The NiCK Study is a cross-sectional cohort study in which neurocognitive and neuroimaging phenotyping is performed in children and young adults, aged 8 to 25 years, with chronic kidney disease compared to healthy controls. Assessments include (1) comprehensive neurocognitive testing (using traditional and computerized methods); (2) detailed clinical phenotyping; and (3) multimodal magnetic resonance imaging (MRI) to assess brain structure (using T1-weighted MRI, T2-weighted MRI, and diffusion tensor imaging), functional connectivity (using functional MRI), and blood flow (using arterial spin labeled MRI). Primary analyses will examine group differences in neurocognitive testing and neuroimaging between subjects with chronic kidney disease and healthy controls. Mechanisms responsible for neurocognitive dysfunction resulting from kidney disease will be explored by examining associations between neurocognitive testing and regional changes in brain structure, functional connectivity, or blood flow. In addition, the neurologic impact of kidney disease comorbidities such as anemia and hypertension will be explored. We highlight aspects of our analytical approach that illustrate the challenges and opportunities posed by data of this scope.
\end{abstract}

Discussion: The NiCK study provides a unique opportunity to address key questions about the biological basis of neurocognitive deficits in chronic kidney disease. Understanding these mechanisms could have great public health impact by guiding screening strategies, delivery of health information, and targeted treatment strategies for chronic kidney disease and its related comorbidities.

Keywords: Neurocognition, Neuropsychological, Chronic kidney disease, Hypertension, Cerebrovascular disease, Cardiovascular disease, Neuroimaging, Magnetic resonance imaging, Children, Adolescents, Adults

\footnotetext{
* Correspondence: furths@email.chop.edu

'Division of Nephrology, Children's Hospital of Philadelphia, 34th and Civic

Center Boulevard, Philadelphia, PA, USA

${ }^{2}$ Department of Pediatrics, Perelman School of Medicine at the University of

Pennsylvania, Philadelphia, PA, USA

Full list of author information is available at the end of the article
}

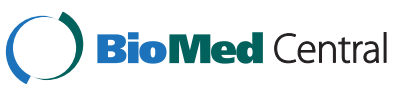

(C) 2015 Hartung et al.; licensee BioMed Central. This is an Open Access article distributed under the terms of the Creative Commons Attribution License (http://creativecommons.org/licenses/by/4.0), which permits unrestricted use, distribution, and reproduction in any medium, provided the original work is properly credited. The Creative Commons Public Domain Dedication waiver (http://creativecommons.org/publicdomain/zero/1.0/) applies to the data made available in this article, unless otherwise stated. 


\section{Background}

Chronic kidney disease (CKD) is strongly linked to neurocognitive dysfunction in adults and children [1-4], but the pathophysiologic processes leading to these deficits remain poorly understood. A number of studies have used neuroimaging to characterize the neuroanatomic changes in children and adults with CKD. A recent systematic review [5] of such studies identified three major types of abnormalities: (1) cerebral atrophy and cerebral density changes; (2) signs of cerebral vascular disease; and (3) regional blood flow changes. While the majority of these studies were performed in adults, the small number of pediatric studies highlighted some overlapping findings, including cerebral atrophy and periventricular white matter infarcts [6-10].

Although these studies have increased awareness of neuroanatomical abnormalities in CKD, a key question remains: What are the specific physiologic disturbances in CKD that lead to neurocognitive changes? Potential mechanisms include metabolic neuronal injury [11] and, perhaps more importantly, subclinical vascular disease [4], mediated through cardiovascular risk factors such as anemia, dyslipidemia, and hypertension. However, it remains unclear how clinical risk factors in CKD produce specific neurophysiologic changes, and how these changes translate to clinically relevant neurocognitive outcomes.

The Neurocognitive Assessment and Magnetic Resonance Imaging Analysis of Children and Young Adults with Chronic Kidney Disease (NiCK) Study was designed to address this critical gap in our understanding of the biological basis for neurologic abnormalities in CKD. With funding from the Pennsylvania Department of Health, this study uses a comprehensive interdisciplinary approach and leverages unique resources at the Children's Hospital of Philadelphia and the University of Pennsylvania to combine comprehensive neurocognitive assessments and stateof-the-art multimodal magnetic resonance imaging (MRI) in children and young adults with CKD.

In this report, we describe the objectives, design, and methods of the NiCK study, and outline how the study provides the opportunity to address key questions in the field. We will then describe our approach to data analysis, focusing on the challenges and opportunities posed by the multitude of variables inherent in clinical, neurocognitive, and neuroimaging data of this scope. Finally, we will outline future directions for this rich dataset, including the potential for novel unbiased scientific discovery, and discuss the potential public health impact of this study.

\section{Objective}

The overarching objectives of the NiCK study are (1) to determine how CKD and its associated comorbidities affect neurocognitive function, and (2) to understand the neurobiological basis for cognitive abnormalities in
CKD. The NiCK study seeks to achieve these objectives using the following three-pronged approach:

1. Detailed neurocognitive description of children and young adults with CKD, along with concurrent age-matched healthy controls, using:

a. a comprehensive battery of traditional neurocognitive testing, and

b. a computerized neurocognitive battery

2. Detailed clinical description to explore the impact of clinical risk factors such as hypertension and anemia; and

3. Multimodal magnetic resonance imaging (MRI) to identify differences in brain structure, functional connectivity, and cerebral blood flow, using:

a. Structural MRI to explore the effects of CKD on brain structure

b. Resting state blood oxygenation level dependent functional MRI (BOLD fMRI) to evaluate connectivity within brain networks that modulate specific neurocognitive functions

c. Arterial spin labeled perfusion MRI to evaluate global and regional cerebral blood flow.

This multifaceted approach will allow us to integrate findings from neurocognitive testing, clinical phenotyping, and multimodal MRI to develop a unique multi-parametric neurocognitive phenotype for CKD. This innovative approach to assessment will provide new knowledge to further our understanding of mechanisms underlying neurological dysfunction in CKD.

\section{Methods/design \\ Study design}

The NiCK study consists of a cross-sectional study of 90 pediatric and young adult subjects, aged $8-25$ years, with Stage 2 to 5 CKD (estimated glomerular filtration rate $[\mathrm{eGFR}]<90 \mathrm{~mL} / \mathrm{min} / 1.73 \mathrm{~m}^{2}$, including dialysis and post-transplant), compared to healthy controls matched on age and socioeconomic status (using insurance status as a proxy).

The study was carried out in accordance with the Declaration of Helsinki and was approved by the Institutional Review Board of the Children's Hospital of Philadelphia. Written informed consent was obtained from all participants or their parents/legal guardians for subjects under age 18 years.

\section{Setting}

The NiCK study is performed at a large tertiary care children's hospital in an urban setting (the Children's Hospital of Philadelphia). 


\section{Study participants}

Table 1 shows the inclusion and exclusion criteria for NiCK study participants. Eligibility for CKD participants is based on eGFR using the bedside CKiD equation [12] for participants aged 8 to 18 years, and the Modification of Diet in Renal Disease (MDRD) study equation [13] for those over 18 years of age. CKD is defined as evidence of kidney dysfunction for more than six months.

The lower age limit of 8 years ensures improved compliance with imaging procedures. Since the selected neurocognitive measures are standardized largely in English, participants must have English as their primary language. To ensure that study results reflect the effects of kidney disease, patients with a number of comorbidities that independently affect brain function (e.g., seizure disorder) or the ability to complete test measures (profound developmental disabilities) are excluded from participating (see Table 1).

\section{Study procedures}

The participant data collected include demographic information (age at visit, age at CKD diagnosis, gender, maternal education, insurance status, race), past medical history, family history, and current medications. Participants then undergo clinical, imaging, and neurocognitive assessments as described below.

\section{Clinical evaluations}

Participants' blood pressure (BP), heart rate, respirations, height, weight, and body mass index are measured. Laboratory data collected includes complete blood count, comprehensive metabolic panel, calcium, phosphate, cystatin C, lipid panel, and urine studies for total protein, albumin, and creatinine. Urine pregnancy testing is performed in post-pubertal girls prior to MRI scanning. All participants undergo 24-hour ambulatory blood pressure monitoring (ABPM).

\section{Neurocognitive assessments Traditional neurocognitive battery}

A battery of age-specific neurocognitive assessments (Table 2) is performed at the baseline visit to measure various aspects of attention/executive functioning via laboratory and parent/participant ratings. In addition, age-specific depression indices and a visual analog anxiety scale are administered. To address the potential effects of fatigue and attention-loss on test performance, the tests are administered in a counterbalanced format across each study participant. A behavior coding mechanism is used to provide examiner perception of the reliability and validity of the test data collected.

\section{Computerized neurocognitive battery}

In addition to the traditional neurocognitive assessments outlined in Table 2, participants also undergo a computerized neurocognitive battery $(\mathrm{CNB})$ developed at the University of Pennsylvania [14]. The Penn CNB includes 14 tests assessing five neurobehavioral functions (Table 3): executive control, episodic memory, complex cognition, social cognition, and praxis speed. The Penn CNB assesses both accuracy (proportion of correct responses) and speed (response time for correct responses), with the latter variable providing an additional area of exploration. The Penn CNB requires approximately one hour to administer. Agespecific norms for the Penn CNB are available based on data from a large cohort $(\mathrm{n} \approx 1800)$ of typically developing

Table 1 Inclusion and exclusion criteria for the NiCK study

\begin{tabular}{|c|c|}
\hline \multirow{3}{*}{$\begin{array}{l}\text { Inclusion } \\
\text { criteria }\end{array}$} & Age 8 - 25 years \\
\hline & $\begin{array}{l}\text { English is primary language for participant (most neurocognitive measures standardized only in English). Parent completing } \\
\text { questionnaires must be proficient in English. }\end{array}$ \\
\hline & Control subjects: Healthy siblings or individuals matched in age and insurance status \\
\hline \multirow{5}{*}{$\begin{array}{l}\text { Exclusion } \\
\text { criteria }\end{array}$} & Conditions that would prohibit MRI: certain types of body metal; claustrophobia \\
\hline & $\begin{array}{l}\text { History of traumatic brain injury or other significant medical or neurological abnormality affecting motor or higher cortical } \\
\text { functioning (e.g. seizure disorder, genetic syndromes, systemic diseases that can affect the brain such as sickle cell disease, cerebral } \\
\text { lupus, spina bifida, gestational age below } 32 \text { weeks, or perinatal injury) }\end{array}$ \\
\hline & $\begin{array}{l}\text { Profound developmental disability or sensory-motor difficulties that would preclude valid use of diagnostic instruments or scanning } \\
\text { procedures. }\end{array}$ \\
\hline & Known drug or alcohol use within 24 hours of any assessment \\
\hline & cause of potential risk of MRI to the unborn fetus). \\
\hline
\end{tabular}


Table 2 Traditional neurocognitive and affective measurements

\begin{tabular}{lll}
\hline Domain & Test name & $\begin{array}{l}\text { Age group for test in this } \\
\text { study }\end{array}$ \\
\hline Intelligence & Wechsler Abbreviated Scales of Intelligence (WASI) & All \\
Attention Regulation & Conners'Continuous Performance Test II (CPT-II) & All \\
& Delis-Kaplan Executive Function System Tower Subtest (D-KEFS Tower) & All \\
Working Memory & Wechsler Intelligence Scale for Children Fourth Edition Integrated (WISC-IV-I) Digit Span & 16 years or younger \\
& Task & 16 years or younger \\
& Wechsler Intelligence Scale for Children Fourth Edition Integrated (WISC-IV-I) Spatial & 17 years or older \\
& Span Task & 17 years or older \\
Wechsler Memory Scale Third Edition (WMS-III) Digit Span Task & 17 years or younger \\
Executive Function & Wechsler Memory Scale Third Edition (WMS-III) Spatial Span Task & 18 years or older \\
Behavior & Behavior Rating Inventory of Executive Function (BRIEF) & 17 years or younger \\
Depression & BRIEF - Adult Version (BRIEF-A) & 18 years or older \\
Anxiety & Children's Depression Inventory II Short (CDI-2 Short) & All
\end{tabular}

children and young adults in the Philadelphia Neurodevelopmental Cohort [15].

\section{Imaging measurements}

After appropriate MRI safety screening, participants undergo non-sedated, non-contrast MRI of the brain, acquired on a Siemens Verio 3 T scanner equipped with a 32channel head coil. The FDA- and manufacturer-approved sequences used are shown in Table 4. The Physiologic Monitoring Unit (PMU) of the Siemens Verio is used to collect pulse oxygenation data during the scan. All images are read by board certified pediatric neuroradiologists, and all clinically significant incidental findings are communicated to the participants.

\section{Structural MRI (sMRI)}

An automated processing pipeline consisting of extensively validated methods is applied for processing sMRI images. The processing pipeline includes: extraction of the brain parenchymal tissue using multi-atlas skullstripping [16]; inhomogeneity correction and tissue segmentation into gray matter (GM), white matter (WM) and cerebrospinal fluid (CSF) [17]; formation of regional volumetric maps, called RAVENS (regional analysis of volumes examined in normalized space) maps [18] using

Table 3 Domains and tests in the Penn computerized neurocognitive battery

\begin{tabular}{|c|c|c|c|}
\hline Neurobehavioral function & Domain & Test name & Label \\
\hline \multirow[t]{3}{*}{ Executive Control } & Abstraction/mental flexibility & Penn Conditional Exclusion Test & PCET \\
\hline & Attention & Penn Continuous Performance Test & PCPT \\
\hline & Working memory & Short Letter N-Back Test & SLNB \\
\hline \multirow[t]{3}{*}{ Episodic Memory } & Verbal Memory & Penn Word Memory & CPW \\
\hline & Facial Memory & Penn Face Memory & CPF \\
\hline & Spatial Memory & Visual Object Learning Test & VOLT \\
\hline \multirow[t]{3}{*}{ Complex Cognition } & Verbal Reasoning & Penn Verbal Reasoning Test & PVRT \\
\hline & Nonverbal Reasoning & Penn Matrix Reasoning Test & PMAT \\
\hline & Spatial Processing & Penn Line Orientation Test & PLOT \\
\hline \multirow[t]{3}{*}{ Social Cognition } & Emotion Identification & Penn Emotion Identification Test & ER40 \\
\hline & Emotion Differentiation & Penn Emotion Differentiation Test & MEDF \\
\hline & Age Differentiation & Penn Age Differentiation Test & ADT \\
\hline \multirow[t]{2}{*}{ Praxis Speed } & Sensorimotor Speed & Motor Praxis & MPRAXIS \\
\hline & Motor Speed & Finger Tapping Test & CTAP \\
\hline
\end{tabular}


Table 4 Magnetic resonance imaging (MRI) Sequences performed in the NiCK study

\begin{tabular}{|c|c|}
\hline Name & Sequence parameters \\
\hline 3D T1 MPRAGE & $\mathrm{TR}=1.79 \mathrm{~s}, \mathrm{TE}=3.06 \mathrm{~ms}, \mathrm{Tl}=1.050 \mathrm{~s}, \mathrm{FoV}=250 \times 250 \mathrm{~mm}^{2}$, flip angle $=10^{\circ}$, voxel size $=1 \times 1 \times 1 \mathrm{~mm}^{3}$ \\
\hline pCASL 2D GE EPI & $\begin{array}{l}\mathrm{TR}=4 \mathrm{~s}, \mathrm{TE}=12 \mathrm{~ms} \text {, FoV } 220 \times 220 \mathrm{~mm}^{2} \text {, flip angle }=90^{\circ} \text {, voxel size }=3.4 \times 3.4 \times 5.0 \mathrm{~mm}^{3} \text {, labeling duration } 1.5 \mathrm{~s} \text {, postlabeling } \\
\text { delay } 1.2 \mathrm{~s}, 40 \mathrm{label} / \text { control pairs }\end{array}$ \\
\hline T2 FLAIR & $\mathrm{TR}=9 \mathrm{~s}, \mathrm{TE}=76 \mathrm{~ms}, \mathrm{Tl}=2.5 \mathrm{~s}$, FoV $=220 \times 220 \mathrm{~mm}^{2}$, flip angle $=146^{\circ}$, voxel size $=0.9 \times 0.9 \times 2 \mathrm{~mm}^{3}$ \\
\hline T1 FLASH & $\mathrm{TR}=300 \mathrm{~ms}, \mathrm{TE}=2.46 \mathrm{~ms}, \mathrm{FoV}=225 \times 225 \mathrm{~mm}^{2}$, flip angle $=60^{\circ}$, voxel size $=0.9 \times 0.9 \times 3 \mathrm{~mm}^{3}$ \\
\hline GRE Field Mapping & $\mathrm{TR}=499 \mathrm{~ms}, \mathrm{TE} 1=5.19 \mathrm{~ms}, \mathrm{TE} 2=7.65 \mathrm{~ms}$, FoV $=192 \times 192 \mathrm{~mm}^{2}$, flip angle $=60^{\circ}$, voxel size $=3 \times 3 \times 3 \mathrm{~mm}^{3}$ \\
\hline $\begin{array}{l}\text { Resting } \mathrm{fMRI} / \mathrm{GE} \\
\text { EPI }\end{array}$ & $\mathrm{TR}=3 \mathrm{~s}$, TE $30 \mathrm{~ms}$, FoV $=192 \times 192 \mathrm{~mm}^{2}$, flip angle $=90^{\circ}$, voxel size $=3 \times 3 \times 3 \mathrm{~mm}^{3}$ isotropic, 102 volumes \\
\hline DTI SE EPI & $\mathrm{TR}=11 \mathrm{~s}, \mathrm{TE}=76.4 \mathrm{~ms}, \mathrm{~b}=0$ and $1000 \mathrm{~s} / \mathrm{mm}^{2}, 30$ directions, FoV $=256 \times 256 \mathrm{~mm}^{2}$, voxel size $=2 \times 2 \times 2 \mathrm{~mm}^{3}$ \\
\hline $\begin{array}{l}\text { Proton Density/T2 } \\
\text { MRI }\end{array}$ & $\mathrm{TR}=6.22 \mathrm{~s}, \mathrm{TE} 1=14 \mathrm{~ms}, \mathrm{TE} 2=98 \mathrm{~ms}$, FoV $=256 \times 256 \mathrm{~mm}^{2}$, flip angle $=150^{\circ}$, voxel size $=1 \times 1 \times 2 \mathrm{~mm}^{3}$ \\
\hline
\end{tabular}

a deformable atlas registration method [19] to enable comparative analysis of tissue volumes in the common template space; segmentation of white matter lesions using a multi-modal supervised learning method [20]; and segmentation into a set of expert-defined anatomic regions of interest (ROIs) using a recently developed multi-atlas label fusion method [21]. The segmentation included 148 ROIs, including 98 cortical regions, white matter regions partitioned into brain lobes, as well as important deep structures such as hippocampus, thalamus and amygdala. The ROIs are organized within a hierarchical structure such that quantitative imaging measurements from normal and abnormal (lesion) tissues could be calculated from different image modalities for both individual ROIs and larger anatomical regions.

By summarizing the very high dimensional image data with much fewer variables, calculated volumetric measurements allow us to perform quantitative analyses of regional brain volumes and fractional anisotropy, and to evaluate the extent of brain atrophy and cerebrovascular disease such as infarcts and leukoareosis.

\section{Blood oxygenation level dependent functional MRI (BOLD fMRI)}

BOLD fMRI is used to evaluate connectivity within specific cognitive networks in the absence of external stimuli (resting state BOLD fMRI). Following standard registration procedures (implemented via the program FIRST) [22], fMRI data are registered to the standard space of the Montreal Neurological Institute (MNI) template. Data analysis focuses primarily on seed-based multiple regression to identify differences in attention networks (for example, the Default Mode Network, or DMN [23]). Multiple regression follows the procedures outlined by Satterthwaite et al. [24], including a variety of nuisance parameters including global fMRI signal, white matter and CSF signal, and head motion. The resulting correlation (r) maps are converted to z-statistic maps (via Fisher r-to-z transformation), which are then used as descriptive statistics for group analyses and per-voxel correlations with clinical and neuropsychological variables.

\section{Arterial spin labeled (ASL) MRI}

ASL MRI uses spin-labeled blood water as an endogenous tracer to measure regional cerebral blood flow (CBF). This study acquired pseudocontinuous ASL [25] sampled with 2-dimensional echoplanar imaging (EPI). ASL images are processed using a perfusion data processing toolbox, ASLtbx [26]. The raw EPI images are first motion-corrected, then pairwise subtraction images are generated from images acquired with and without arterial labeling. The averaged difference images are used to calculate CBF (in $\mathrm{mL} / 100 \mathrm{~g} / \mathrm{min}$ ) as described by Wang et al. [27]. Each subject's data are then segmented into GM, WM, whole-brain CBF maps that are used for group comparisons or correlations with clinical or neurocognitive indices. The ASL images are also normalized to the standard space of the MNI brain template for voxel-based analyses.

\section{Study size}

A sample size of 90 CKD subjects and 90 healthy controls was calculated based on the assumption that the prevalence of MRI abnormalities in CKD subjects will be $12.5 \%$ and will be $1 \%$ in normal controls. This sample size provides $80 \%$ power to detect such a difference with 95\% confidence.

\section{Key questions addressed by this study}

The comprehensive approach employed in the NiCK study provides the opportunity to address some key issues and challenges in the field. 


\section{How do cardiovascular risk factors contribute to neurocognitive dysfunction in CKD, and does CKD affect neurocognition independent of its vascular effects?}

Vascular disease is a well-known independent risk factor for cognitive impairment and dementia in adults [28]. Adults with CKD are at increased risk for stroke, silent brain infarcts, white matter lesions, and dementia, presumably due to cerebral small-vessel disease [4]. However, the extent to which CKD affects neurocognition independently of its vascular effects is unclear [29]. Discerning a CKD-specific effect is difficult in adults due to the high prevalence of pre-existing cardiovascular disease. In contrast, children with CKD generally have fewer pre-existing cardiovascular comorbidities. Studying children and young adults with CKD therefore provides the opportunity to identify neurologic changes that may be specific to CKD.

By comparing neurocognitive test results with MRI data, we will be able to evaluate whether specific deficits correlate with changes in structure, connectivity, or $\mathrm{CBF}$ in particular regions of the brain. For example, executive function is a domain known to be affected in children with CKD [3]. We would therefore hypothesize that children with deficits in executive function may have abnormalities in the prefrontal cortex and frontotemporal regions, such as white matter changes or volume loss on sMRI, or regional CBF changes on ASL MRI. The impact of level of kidney function on specific imaging parameters can be evaluated. In addition, the effect of cardiovascular risk factors can be analyzed, such as hypertension, anemia, dyslipidemia, and calcium-phosphate product. Examining these changes in children and young adults may help to elucidate targets for early intervention.

\section{By what mechanisms do risk factors such as hypertension contribute to neurocognitive deficits in CKD?}

Hypertension has been associated with neurocognitive dysfunction in adults [30-32] and children [33,34] with and without CKD. Possible mechanisms include vascular remodeling, altered cerebrovascular reactivity, or direct neuronal effects [35-37], but there are few studies that directly explore the neurophysiologic impact of hypertension [30-32]. The NiCK study provides a unique opportunity to address this gap in knowledge by combining detailed clinical phenotyping including casual BP measurements and ABPM, with comprehensive neurocognitive testing and multimodal MRI. This strategy allows us to identify specific neurocognitive deficits caused by hypertension, and directly correlate them with global or regional brain abnormalities such as changes in CBF on ASL MRI, or changes in functional connectivity on resting state BOLD fMRI.

\section{How does CKD affect neurocognitive function and structure at different stages of development?}

Brain maturation involves a complex series of structural and functional changes throughout childhood, adolescence, and young adulthood [38]. By studying cognitive function and brain structure in a cross-sectional sample of participants aged 8-25 years with and without CKD, the NiCK study will allow us to explore how CKD and its related comorbidities affect neurodevelopment at different ages. This may allow us to identify targets for early screening or therapeutic intervention.

\section{Approaches to data analyses and challenges}

The data collected in the NiCK study provides great opportunities to test hypotheses regarding differences in brain structure and function between individuals with CKD and healthy controls. However, the heterogeneous nature of CKD participants in this study presents both challenges and opportunities. Their wide range of kidney function (from dialysis-dependent up to eGFR $90 \mathrm{~mL} /$ $\mathrm{min} / 1.73 \mathrm{~m}^{2}$ ) allows us to explore neurologic changes across the continuum of disease severity. However, this diversity also introduces a greater number of covariates that must be accounted for in our analyses.

Analysis of the large number of clinical and imaging variables also presents significant challenges, particularly in developing strategies to handle multiple comparisons. For example, each neurocognitive testing method involves numerous individual tasks and scores; ABPM produces dozens of summary measures; and each MRI image consists of millions of voxels. The analysis plan for this study therefore includes a number of strategies for data reduction and for comparisons of measures across various modalities.

\section{Clinical variables \\ Kidney disease severity}

To determine the effect of CKD on neurocognitive function and neuroimaging parameters, our primary analytic approach will be group comparisons between CKD and control subjects. However, it is important to recognize that the wide range of kidney function in the CKD group could obscure effects of more severe kidney disease on neurocognitive function; consequently, other measures of CKD severity need to be considered. Further, while eGFR would seem to be a logical marker of kidney disease severity, this study includes post-transplant subjects and current eGFR may not account for other elements of disease trajectory that could affect neurodevelopment. To try to overcome this limitation, we devised a Severity Score (Table 5) to use in our analyses. Based on an additive cumulative risk model, this score includes additional indicators of past disease activity - whether the subject has ever required dialysis or transplant, and whether 
Table 5 Severity score system

\begin{tabular}{|c|c|}
\hline Clinical characteristics & Points \\
\hline \multicolumn{2}{|l|}{ Past disease activity score } \\
\hline Has the subject ever received dialysis? & $\begin{array}{l}\text { No }=0, \\
\text { Yes }=1\end{array}$ \\
\hline Has the subject ever received a kidney transplant? & $\begin{array}{l}\text { No }=0, \\
\text { Yes }=1\end{array}$ \\
\hline $\begin{array}{l}\text { Did the subject receive dialysis or transplant at age } \\
\leq 5 \text { years? }\end{array}$ & $\begin{array}{l}\text { No }=0 \\
\text { Yes }=1\end{array}$ \\
\hline \multicolumn{2}{|l|}{$\begin{array}{l}\text { Current estimated glomerular filtration rate } \\
\left(\text { eGFR, } \mathrm{ml} / \mathrm{min} / 1.73 \mathrm{~m}^{2}\right) \text { score }\end{array}$} \\
\hline eGFR $>60$ & 0 \\
\hline $30<\mathrm{eGFR} \leq 60$ & 1 \\
\hline $15<\mathrm{eGFR} \leq 30$ & 2 \\
\hline $\mathrm{eGFR} \leq 15$ & 3 \\
\hline $\begin{array}{l}\text { Severity Score (past disease activity score + } \\
\text { current eGFR score) }\end{array}$ & 0 to 6 \\
\hline
\end{tabular}

eGFR, estimated glomerular filtration rate (using the bedside CKiD equation [12] for subjects aged $<18$ years or the MDRD equation [13] for those $\geq 18$ years).

renal replacement therapy was required at age $\leq 5$ years, a critical developmental window. These factors are combined with a score for eGFR groupings, to result in a final Severity Score that ranges from 0 (least severe) to 6 (most severe). The Severity Score approach is simple, and by virtue of being based on objectively determined criteria, it is not subject to inter-rater variability. The additional information gained by the Severity Score is illustrated in Figure 1, which represents the clinical course of two hypothetical subjects of the same age with the same eGFR at the time of the study visit. Despite the similarity in their current kidney function, it is clear that subject B's trajectory has the potential to inflict greater neurological and neurocognitive insult compared to that of subject A - this is reflected in a higher Severity Score ( 5 for subject B vs. 2 for subject A). Use of the Severity Score in our analyses therefore allows us to include these additional factors that could affect neurodevelopmental outcome, and contributes to data reduction.

\section{Ambulatory blood pressure monitoring}

There are a large number of blood pressure (BP) variables generated by 24-hour ABPM. These include measures for systolic and diastolic BP (SBP and DBP) and heart rate (HR), and can be broken down into waking hours, sleeping hours, and the total 24 hours. Potential variables include mean BP, mean BP index (BP normalized for age, height, and gender), BP load (proportion of readings exceeding the 95th percentile for age, height, and gender), nocturnal BP dipping (percent decrease in mean BP from waking to sleeping), hyperbaric index (area enclosed by the line of ambulatory BP readings above and the 95th percentile limit line for BP readings below), and BP and $\mathrm{HR}$ variability (coefficient of variation of $\mathrm{BP}$ readings and HR). Many of these variables are highly correlated with each other (e.g. mean BP and BP load); however, a growing number of studies show that certain ABPM variables, for example abnormal nocturnal dipping or increased BP variability, are independent predictors of adverse cardiovascular and neurocognitive outcomes. Our approach in selecting which ABPM variables to focus our analyses on has been two-pronged: (1) review of the literature to create an a priori list of variables expected to have the greatest clinical significance, and (2) assessment for correlation between ABPM variables - the variables chosen for our

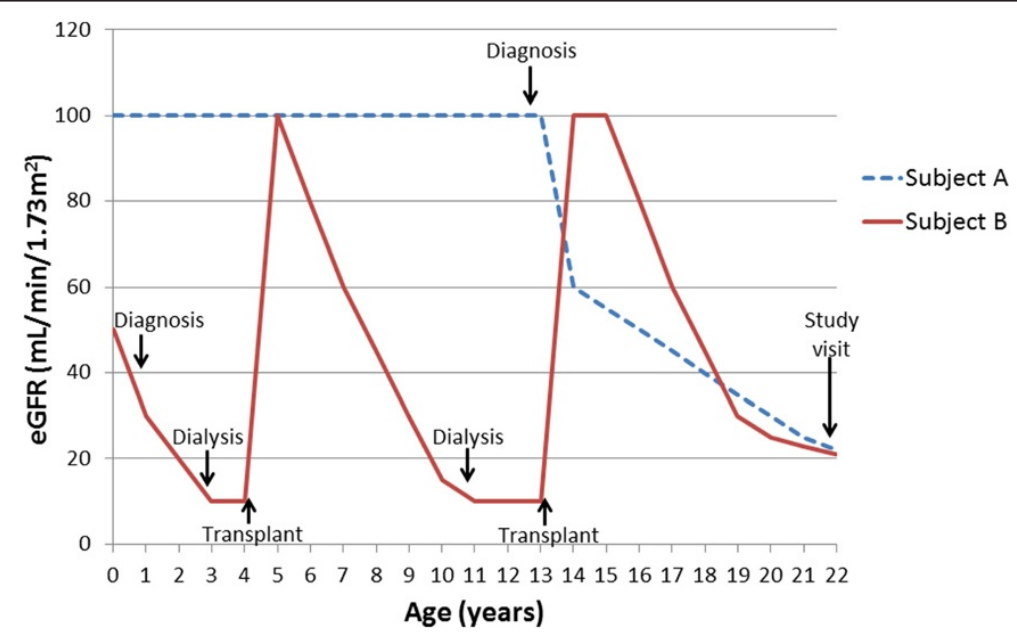

Figure 1 Illustration of the value of the severity score.Trajectory of estimated glomerular filtration rate (eGFR) of two hypothetical subjects of the same age with the same eGFR at the time of the study visit. Despite the similarity in their current kidney function, subject B's course may have inflicted greater neurocognitive insult compared to that of subject A. This difference in clinical course is reflected in their Severity Scores (5 for subject B vs. 2 for subject A - see Table 5), but would be missed if we only considered current eGFR. 
primary analyses were those that were not highly correlated with each other, and thus potentially most informative about different aspects of BP physiology.

\section{Neurocognitive assessments}

This study includes comprehensive neurocognitive phenotyping in youth with CKD. Although traditional neurocognitive batteries (TNB) have been used extensively in individuals with CKD, this is the first study to utilize a computerized testing (the Penn CNB) in this population. Both the TNB and CNB provide comprehensive assessments of multiple neurocognitive domains. However, since the exact tests differ between the two batteries, one challenge we encounter is aligning specific tasks in each battery with neurocognitive functions tested. Table 6 demonstrates the framework used in this study for mapping tests within the TNB and CNB to various neurocognitive domains. As the table illustrates, some neurocognitive domains are tested by multiple components of the TNB and CNB. At the same time, some tests can span several neurocognitive domains (for example, the Penn Verbal Reasoning Test [PVRT] assesses both language

Table 6 Framework for mapping tests/tasks in the traditional and computerized neurocognitive batteries to specific neurocognitive domains

\begin{tabular}{|c|c|c|}
\hline Domain & Traditional neurocognitive battery (TNB) tests & $\begin{array}{l}\text { Computerized neurocognitive battery } \\
\text { (CNB) tests }\end{array}$ \\
\hline \multirow[t]{2}{*}{ Language } & WASI Vocabulary Subtest T-Score & \multirow[t]{2}{*}{ Language Reasoning (PVRT) } \\
\hline & WASI Similarities Subtest T-Score & \\
\hline \multirow[t]{6}{*}{ Attention } & $\begin{array}{l}\text { WMS-III Digit Span Forward Scaled Score/WISC-IV-I Digit Span Forward } \\
\text { Scaled Score }\end{array}$ & \multirow[t]{6}{*}{ Attention (PCPT) } \\
\hline & $\begin{array}{l}\text { WMS-III Spatial Span Forward Scaled Score/ WISC-IV-I Spatial Span Forward } \\
\text { Scaled Score }\end{array}$ & \\
\hline & CPT-II Omissions T-Score & \\
\hline & CPT-II Variability T-Score & \\
\hline & CPT-II Detectability T-Score & \\
\hline & CPT-II Response Style T-Score & \\
\hline \multirow[t]{3}{*}{ Inhibitory Control } & \multirow[t]{2}{*}{ CPT-II Commissions T-Score } & False Positive (i.e. incorrect) scores from: \\
\hline & & Working Memory (SLNB) \\
\hline & CPT-II Hit RT T-Score & Attention (PCPT) \\
\hline \multirow[t]{3}{*}{ Planned problem solving } & WASI Matrix Reasoning Subtest T Score & Nonverbal Reasoning (PMAT) \\
\hline & D-KEFS Total Achievement Scaled Score & \multirow[t]{2}{*}{ Language Reasoning (PVRT) } \\
\hline & D-KEFS Mean First-Move Time Scaled Score & \\
\hline Set Shifting & D-KEFS Move Accuracy Ratio Scaled Score & Abstraction/Mental Flexibility (PCET) \\
\hline \multirow[t]{2}{*}{ Visual Spatial } & WASI Block Design Subtest T Score & Nonverbal Reasoning (PMAT) \\
\hline & WASI Matrix Reasoning Subtest T Score & Spatial Processing (PLOT) \\
\hline Verbal Working Memory & $\begin{array}{l}\text { WMS-III Digit Span Backward Scaled Score/WISC-IV-I Digit Span Backward } \\
\text { Scaled Score }\end{array}$ & Working Memory (SLNB) \\
\hline Visual Working Memory & $\begin{array}{l}\text { WMS-III Spatial Span Backward Scaled Score/WISC-IV-I Spatial Span } \\
\text { Backward Scaled Score }\end{array}$ & Working Memory (SLNB) \\
\hline Verbal Memory & $\begin{array}{l}\text { WMS-III Digit Span Forward Scaled Score/WISC-IV-I Digit Span Forward } \\
\text { Scaled Score }\end{array}$ & Verbal Memory (CPW) \\
\hline \multirow[t]{2}{*}{ Visual Memory } & \multirow{2}{*}{$\begin{array}{l}\text { WMS-III Spatial Span Forward Scaled Score/ WISC-IV-I Spatial Span Forward } \\
\text { Scaled Score }\end{array}$} & Facial Memory (CPF) \\
\hline & & Spatial Memory (VOLT) \\
\hline $\begin{array}{l}\text { Ratings of Executive } \\
\text { Function }\end{array}$ & Global Executive Composite & Abstraction/Mental Flexibility (PCET) \\
\hline \multirow[t]{3}{*}{ Social Cognition } & \multirow[t]{3}{*}{ None } & Emotion Identification (ER40) \\
\hline & & Emotion Differentiation (MEDF) \\
\hline & & Age Differentiation (ADT) \\
\hline \multirow[t]{2}{*}{ Motor Speed } & \multirow[t]{2}{*}{ CPT-II Hit RT T-Score } & Sensorimotor Speed (MPRAXIS) \\
\hline & & Motor Speed (CTAP) \\
\hline
\end{tabular}


and planned problem solving). Since performance across various tests and domains can be highly correlated, approaches such as factor analysis and principal component analysis are required in our analyses.

Interpretation of performance across the TNB and CNB presents another challenge. Most TNB tests measure only accuracy, whereas most CNB tests measure both accuracy and speed. This is potentially important, as recent studies have shown that deviations from normal brain developmental trajectories are associated with significant deviations in performance for speed, but not accuracy [38]. For some domains (for example, attention), improved overall performance is reflected by better accuracy as well as faster speed. In contrast, in tasks that require more complex problem-solving (for example, PVRT and PMAT), we may see divergence in performance for accuracy and speed. Therefore, different analytic approaches may be required to interpret TNB and $\mathrm{CNB}$ performances across various domains. For example, for some analyses we plan to analyze accuracy separately, but with associated adjustments for speed.

\section{Imaging assessments}

Our primary approach for analysis of sMRI, fMRI, and ASL MRI data in the NiCK study is to compare group differences in pre-specified ROIs, based on anatomic partitioning into structural/functional brain regions.

Using these pre-specified ROIs, we can explore specific hypotheses - for example, ROIs encompassing the prefrontal cortex and frontotemporal areas would be hypothesized to be affected by CKD, given literature describing deficits in executive function in this population [3]. We can therefore use the multimodal imaging approaches to compare characteristics of CKD and control subjects in these ROIs, using sMRI to detect volumetric differences, BOLD fMRI to test for differences in functional connectivity, and ASL MRI to compare regional CBF differences.

Whole brain approaches can also be used to explore the global effects of specific CKD comorbidities - for example, using ASL, we can examine the effects of hypertension and anemia on global cerebral blood flow (CBF), or CBF within gray matter and white matter compartments.

Additionally, a priori correlations with the neurocognitive data will be explored in an effort to identify underlying neurological processes for manifest neurocognitive dysfunction.

\section{Future directions}

Although the primary analyses in $\mathrm{NiCK}$ will be hypothesis-driven, the nature of the imaging data collected also provides the opportunity to explore potential new connections and generate new hypotheses. For example, one hypothesis-free approach to analysis of the sMRI data uses voxel-based analysis [18,39] - this method evaluates the image data at its full resolution without a priori partitioning into anatomical ROIs. This unbiased discovery approach has the potential to yield new, previously unsuspected connections between regional brain abnormalities and CKD comorbidities.

A new machine learning methodology, the brain development index (BDI) [38], summarizes the multivariate pattern of structural brain development using a large sample of subjects ages 8-22, and can accurately delineate trajectories of brain development. Calculating and analyzing the BDI for NiCK subjects may potentially help us for identification of subtle developmental abnormalities related to CKD.

The long-term goal of the NiCK study is to develop a multi-parametric neuroimaging biomarker for patients with CKD. Using machine learning and pattern recognition methods, a classification system incorporating all the imaging measurements can be developed for individual patients. Multivariate pattern classification combining signals from SMRI, ASL and fMRI can be performed using the COMPARE (classification of morphological patterns using adaptive regional elements) [40] method. This combines signals from all imaging modalities to determine the set of brain regions and measurements that jointly offer the most distinctive set of measurements that characterizes the brain of CKD patients. In future studies, this sort of multi-parametric imaging index could be evaluated as a prognostic indicator to assess whether imaging findings can predict subsequent neurocognitive decline.

\section{Discussion}

The NiCK Study represents the most comprehensive neurocognitive and neuroimaging phenotyping ever performed in children and youth with CKD. Our innovative approach of integrating multimodal MRI with neurocognitive and clinical data provides a unique opportunity to answer key questions in the field. These include understanding the independent neurologic impact of CKD, the neurologic effects of hypertension and other cardiovascular risk factors, and the consequences of CKD on neurodevelopment from childhood through young adulthood. Understanding these mechanisms could have great potential public health impact. First, this study could help to identify improved screening strategies to identify patients at high risk of adverse neurocognitive outcomes (for example, determining whether neuropsychological testing or neuroimaging should be incorporated into clinical care of youth with CKD). Second, neurocognitive impairment may affect adherence to the complex medical regimens that are routinely prescribed to patients with CKD. By improving our recognition of these deficits in our patients, we may be able to deliver health information in a more individualized and effective manner. Finally, by identifying mechanisms through which modifiable risk factors such as 
anemia and hypertension affect neurocognition, this study may help to guide targeted treatment strategies to prevent future cognitive decline.

\section{Abbreviations}

ABPM: Ambulatory blood pressure monitoring; ASL MRI: Arterial spin labeled magnetic resonance perfusion imaging; BOLD fMRI: Blood oxygenation level dependent functional magnetic resonance imaging; BP: Blood pressure; CBF: Cerebral blood flow; CKD: Chronic kidney disease; CNB: Computerized neurocognitive battery; DBP: Diastolic blood pressure; eGFR: Estimated glomerular filtration rate; HR: Heart rate; MDRD: Modification of Diet in Renal Disease study; MRI: Magnetic resonance imaging; NiCK: Neurocognitive Assessment and Magnetic Resonance Imaging Analysis of Children and Young Adults with Chronic Kidney Disease Study; ROl: Region of interest; SBP: Systolic blood pressure; sMRI: Structural MRI; TNB: Traditional neurocognitive battery.

\section{Competing interests}

The authors declare that they have no competing interests.

\section{Authors' contributions}

EAH drafted the manuscript, and participated in data collection and analysis. $\mathrm{NL}$ is the study coordinator and is responsible for patient recruitment, data collection and management. JYK and AFJ are biostatisticians responsible for data management and analyses. RLR, DGM, and AMP participated in data collection and analysis. JAD, CD, RTS, RCG, JR, and SRH participated in study design, data management, and data analysis. HSL, GE, JJD, and JDH performed data analysis. SLF is study Principal Investigator; she held primary responsibility for study design and conduct. All authors reviewed and edited the manuscript, and approved the final version.

\section{Acknowledgements}

This project is funded, in part, under a Commonwealth Universal Research Enhancement grant with the Pennsylvania Department of Health, \# SAP 4100054843. The Department specifically disclaims responsibility for any analyses, interpretations or conclusions.

Dr. Hartung is supported by the National Center for Advancing Translational Sciences (NCATS) of the National Institutes of Health $(\mathrm{NIH})$ under Award Number KL2TR000139. The content is solely the responsibility of the authors and does not necessarily represent the official view of NCATS or the NIH. Study data were collected and managed using REDCap electronic data capture tools hosted at The Children's Hospital of Philadelphia. REDCap (Research Electronic Data Capture) [41] is a secure, web-based application designed to support data capture for research studies, providing 1) an intuitive interface for validated data entry; 2) audit trails for tracking data manipulation and export procedures; 3 ) automated export procedures for seamless data downloads to common statistical packages; and 4) procedures for importing data from external sources.

The Clinical and Translational Research Center at the Children's Hospital of Philadelphia is supported by the National Center for Research Resources and the National Center for Advancing Translational Sciences, National Institutes of Health, through Grants UL1RR024134 and UL1TR000003. The content is solely the responsibility of the authors and does not necessarily represent the official views of the $\mathrm{NIH}$.

We would like to thank the patients and families who have participated in the study.

\section{Author details}

'Division of Nephrology, Children's Hospital of Philadelphia, 34th and Civic Center Boulevard, Philadelphia, PA, USA. ${ }^{2}$ Department of Pediatrics, Perelman School of Medicine at the University of Pennsylvania, Philadelphia, PA, USA. ${ }^{3}$ Biostatistics Core, Clinical and Translational Research Center, Children's Hospital of Philadelphia, Philadelphia, PA, USA. ${ }^{4}$ Department of Neurology, Perelman School of Medicine at the University of Pennsylvania, Philadelphia, PA, USA. ${ }^{5}$ Graduate Institute of Clinical Medicine and Imaging Research Center, College of Medicine, Taipei Medical University, Taipei, Taiwan. ${ }^{6}$ Department of Medical Imaging, Taipei Medical University Hospital, Taipei, Taiwan. ${ }^{7}$ Center for Biomedical Image Computing and Analytics, Department of Radiology, Perelman School of Medicine at the University of Pennsylvania, Philadelphia, PA, USA. ${ }^{8}$ Center for Autism Research, Children's Hospital of Philadelphia, Philadelphia, PA, USA. ${ }^{9}$ Department of Psychiatry, Perelman
School of Medicine at the University of Pennsylvania, Philadelphia, PA, USA. ${ }^{10}$ Children's Hospital of Philadelphia, Philadelphia, PA, USA. ${ }^{11}$ Division of Pediatric Nephrology, Department of Pediatrics, Nemours/Alfred I. duPont Hospital for Children, Wilmington, DE, USA. ${ }^{12}$ Brain and Behavior Laboratory, Department of Psychiatry, University of Pennsylvania, Philadelphia, PA, USA.

${ }^{13}$ Division of Developmental and Behavioral Pediatrics, Children's Hospital of Philadelphia, Philadelphia, PA, USA. ${ }^{14}$ Department of Allied Health Sciences, University of North Carolina School of Medicine, Chapel Hill, NC, USA.

${ }^{15}$ Department of Epidemiology, Perelman School of Medicine at the University of Pennsylvania, Philadelphia, PA, USA.

Received: 23 February 2015 Accepted: 22 April 2015 Published online: 30 April 2015

\section{References}

1. Gerson AC, Butler R, Moxey-Mims M, Wentz A, Shinnar S, Lande MB, et al. Neurocognitive outcomes in children with chronic kidney disease: current findings and contemporary endeavors. Ment Retard Dev Disabil Res Rev. 2006;12:208-15.

2. Hooper SR, Gerson AC, Butler RW, Gipson DS, Mendley SR, Lande MB, et al. Neurocognitive functioning of children and adolescents with mild-tomoderate chronic kidney disease. Clin J Am Soc Nephrol. 2011;6:1824-30.

3. Gipson DS, Hooper SR, Duquette PJ, Wetherington CE, Stellwagen KK, Jenkins TL, et al. Memory and executive functions in pediatric chronic kidney disease. Child Neuropsychol. 2006;12:391-405.

4. Bugnicourt J-M, Godefroy O, Chillon J-M, Choukroun G, Massy ZA. Cognitive disorders and dementia in CKD: the neglected kidney-brain axis. J Am Soc Nephrol. 2013;24:353-63.

5. Moodalbail DG, Reiser KA, Detre JA, Schultz RT, Herrington JD, Davatzikos C, et al. Systematic review of structural and functional neuroimaging findings in children and adults with CKD. Clin J Am Soc Nephrol. 2013;8:1429-48.

6. Schnaper HW, Cole BR, Hodges FJ, Robson AM. Cerebral cortical atrophy in pediatric patients with end-stage renal disease. Am J Kidney Dis. 1983;2:645-50.

7. Steinberg A, Efrat R, Pomeranz A, Drukker A. Computerized tomography of the brain in children with chronic renal failure. Int J Pediatr Nephrol. 1985;6:121-6.

8. Elzouki A, Carroll J, Butinar D, Moosa A. Improved neurological outcome in children with chronic renal disease from infancy. Pediatr Nephrol. 1994;8:205-10.

9. Valanne L, Qvist E, Jalanko H, Holmberg C, Pihko H. Neuroradiologic findings in children with renal transplantation under 5 years of age. Pediatr Transplant. 2004;8:44-51.

10. Gipson DS, Duquette PJ, Icard PF, Hooper SR. The central nervous system in childhood chronic kidney disease. Pediatr Nephrol. 2007;22:1703-10.

11. Tryc AB, Alwan G, Bokemeyer M, Goldbecker A, Hecker H, Haubitz M, et al. Cerebral metabolic alterations and cognitive dysfunction in chronic kidney disease. Nephrol Dial Transplant. 2011;26:2635-41.

12. Schwartz GJ, Munoz A, Schneider MF, Mak RH, Kaskel F, Warady BA, et al. New equations to estimate GFR in children with CKD. J Am Soc Nephrol. 2009;20:629-37.

13. Levey AS, Coresh J, Greene T, Stevens LA, Zhang YL, Hendriksen S, et al. Using standardized serum creatinine values in the modification of diet in renal disease study equation for estimating glomerular filtration rate. Ann Intern Med. 2006;145:247-54.

14. Gur RC, Richard J, Hughett P, Calkins ME, Macy L, Bilker WB, et al. A cognitive neuroscience-based computerized battery for efficient measurement of individual differences: standardization and initial construct validation. J Neurosci Methods. 2010;187:254-62.

15. Gur RC, Richard J, Calkins ME, Chiavacci R, Hansen JA, Bilker WB, et al. Age group and sex differences in performance on a computerized neurocognitive battery in children age 8-21. Neuropsychology. 2012;26:251-65.

16. Doshi J, Erus G, Ou Y, Gaonkar B, Davatzikos C. Multi-atlas skull-stripping. Acad Radiol. 2013;20:1566-76.

17. Li C, Gore JC, Davatzikos C. Multiplicative intrinsic component optimization (MICO) for MRI bias field estimation and tissue segmentation. Magn Reson Imaging. 2014;32:913-23.

18. Davatzikos C, Genc A, Xu D, Resnick SM. Voxel-based morphometry using the RAVENS maps: methods and validation using simulated longitudinal atrophy. Neuroimage. 2001;14:1361-9.

19. Ou Y, Sotiras A, Paragios N, Davatzikos C. DRAMMS: Deformable registration via attribute matching and mutual-saliency weighting. Med Image Anal. 2011;15:622-39. 
20. Zacharaki El, Kanterakis S, Bryan RN, Davatzikos C. Measuring brain lesion progression with a supervised tissue classification system. Med Image Comput Comput Assist Interv. 2008;11(Pt 1):620-7.

21. Doshi JJ, Erus G, Ou Y, Davatzikos C. Ensemble-based medical image labeling via sampling morphological appearance manifolds. In: MICCAI Challenge Workshop on Segmentation: Algorithms, Theory and Applications. Nagoya, Japan; 2013. Available at: https://masi.vuse.vanderbilt.edu/workshop2013/ index.php/Program_and_Proceedings.

22. Patenaude B, Smith SM, Kennedy DN, Jenkinson M. A Bayesian model of shape and appearance for subcortical brain segmentation. Neuroimage. 2011;56:907-22.

23. Fox MD, Snyder AZ, Vincent JL, Corbetta M, Van Essen DC, Raichle ME. The human brain is intrinsically organized into dynamic, anticorrelated functional networks. Proc Natl Acad Sci U S A. 2005;102:9673-8.

24. Satterthwaite TD, Wolf DH, Loughead J, Ruparel K, Elliott MA, Hakonarson H, et al. Impact of in-scanner head motion on multiple measures of functional connectivity: relevance for studies of neurodevelopment in youth. Neuroimage. 2012;60:623-32.

25. Dai W, Garcia D, de Bazelaire C, Alsop DC. Continuous flow-driven inversion for arterial spin labeling using pulsed radio frequency and gradient fields. Magn Reson Med. 2008;60:1488-97.

26. Wang Z, Aguirre GK, Rao H, Wang J, Fernández-Seara MA, Childress AR, et al. Empirical optimization of ASL data analysis using an ASL data processing toolbox: ASLtbx. Magn Reson Imaging. 2008;26:261-9.

27. Wang J, Alsop DC, Song HK, Maldjian JA, Tang K, Salvucci AE, et al. Arterial transit time imaging with flow encoding arterial spin tagging (FEAST). Magn Reson Med. 2003;50:599-607.

28. Gorelick PB, Scuteri A, Black SE, Decarli C, Greenberg SM, ladecola C, et al. Vascular contributions to cognitive impairment and dementia: a statement for healthcare professionals from the american heart association/american stroke association. Stroke. 2011:42:2672-713.

29. Miwa K, Tanaka M, Okazaki S, Furukado S, Yagita Y, Sakaguchi M, et al. Chronic kidney disease is associated with dementia independent of cerebral small-vessel disease. Neurology. 2014;82:1051-7.

30. Vicario A, Martinez CD, Baretto D, Diaz Casale A, Nicolosi L. Hypertension and cognitive decline: impact on executive function. J Clin Hypertens (Greenwich). 2005:7:598-604.

31. Waldstein SR. Hypertension and neuropsychological function: a lifespan perspective. Exp Aging Res. 1995;21:321-52.

32. Launer LJ, Ross GW, Petrovitch H, Masaki K, Foley D, White LR, et al. Midlife blood pressure and dementia: the Honolulu-Asia aging study. Neurobiol Aging. 2000;21:49-55.

33. Lande MB, Kupferman JC, Adams HR. Neurocognitive alterations in hypertensive children and adolescents. J Clin Hypertens (Greenwich). 2012;14:353-9.

34. Kupferman JC, Lande MB, Adams HR, Pavlakis SG. Primary hypertension and neurocognitive and executive functioning in school-age children. Pediatr Nephrol. 2013;28:401-8.

35. Jennings JR. Autoregulation of blood pressure and thought: preliminary results of an application of brain imaging to psychosomatic medicine. Psychosom Med. 2003;65:384-95.

36. Jennings JR, Zanstra $Y$. Is the brain the essential in hypertension? Neuroimage. 2009;47:914-21.

37. Ostrovskaya MA, Rojas M, Kupferman JC, Lande MB, Paterno K, Brosgol Y et al. Executive Function and Cerebrovascular Reactivity in Pediatric Hypertension. J Child Neurol. 2015;30:543-6.

38. Erus G, Battapady H, Satterthwaite TD, Hakonarson H, Gur RE, Davatzikos C, et al. Imaging Patterns of Brain Development and their Relationship to Cognition. Cereb Cortex 2014 [Epub ahead of print]

39. Zhang T, Davatzikos C. ODVBA: optimally-discriminative voxel-based analysis. IEEE Trans Med Imaging. 2011;30:1441-54.

40. Fan Y, Shen D, Gur RC, Gur RE, Davatzikos C. COMPARE: classification of morphological patterns using adaptive regional elements. IEEE Trans Med Imaging. 2007:26:93-105

41. Harris PA, Taylor R, Thielke R, Payne J, Gonzalez N, Conde JG. Research electronic data capture (REDCap)-a metadata-driven methodology and workflow process for providing translational research informatics support. J Biomed Inform. 2009;42:377-81.

\section{Submit your next manuscript to BioMed Central and take full advantage of:}

- Convenient online submission

- Thorough peer review

- No space constraints or color figure charges

- Immediate publication on acceptance

- Inclusion in PubMed, CAS, Scopus and Google Scholar

- Research which is freely available for redistribution

Submit your manuscript at www.biomedcentral.com/submit 